For numbered affiliations see end of article.

Correspondence to: Francois Lamontagne

francois.lamontagne@usherbrooke.ca, or Miriam Stegemann miriam.stegemann@charite.de Additional material is published online only. To view please visit the journal online

Cite this as: BMJ 2021;372:n526 http://dx.doi.org/10.1136/bmj.n526

RAPID RECOMMENDATIONS

\title{
A living WHO guideline on drugs to prevent covid-19
}

François Lamontagne, ${ }^{1}$ Miriam Stegemann, ${ }^{2}$ Arnav Agarwal, ${ }^{3}$ Thomas Agoritsas, ${ }^{4,5,6,{ }^{*}}$ Reed Siemieniuk, ${ }^{5}$ 7, ${ }^{*}$ Bram Rochwerg, ${ }^{5,7,{ }^{*}}$ Jessica Bartoszko, ${ }^{5,{ }^{*}}$ Lisa Askie, ${ }^{8,{ }^{*}}$ Helen Macdonald, ${ }^{9,}{ }^{*}$ Muna Al-Maslamani, ${ }^{10}$ Wagdy Amin, ${ }^{11}$ Andre Ricardo Araujo Da Silva, ${ }^{12}$ Fabian Alberto Jaimes Barragan, ${ }^{13}$

Frederique Jacquerioz Bausch, ${ }^{14}$ Erlina Burhan, ${ }^{15}$ Maurizio Cecconi, ${ }^{16}$ Binila Chacko, ${ }^{17}$ Duncan Chanda, ${ }^{18}$ Vu Quoc Dat, ${ }^{19}$ Bin Du, ${ }^{20}$ Heike Geduld, ${ }^{21}$ Patrick Gee, ${ }^{22}$ Muhammad Haider, ${ }^{23}$ Nerina Harley, ${ }^{24}$ Madiha Hashimi, ${ }^{25}$ Fyezah Jehan, ${ }^{26}$ David Hui, ${ }^{27}$ Beverley J Hunt, ${ }^{28}$ Mohamed Ismail, ${ }^{29}$ Sushil Kabra, ${ }^{30}$ Seema Kanda, ${ }^{31}$ Leticia Kawano-Dourado, ${ }^{32}$ Yae-Jean Kim, ${ }^{33}$ Niranjan Kissoon, ${ }^{34}$ Sanjeev Krishna, ${ }^{35}$ Arthur Kwizera, ${ }^{36}$ Thiago Lisboa, ${ }^{37}$ Yee-Sin Leo, ${ }^{38}$ Imelda Mahaka, ${ }^{39}$ Manai Hela, ${ }^{40}$ Giovanni Battista Migliori, ${ }^{41}$ Greta Mino, ${ }^{42}$ Emmanuel Nsutebu, ${ }^{43}$ Natalia Pshenichnaya, ${ }^{44}$ Nida Qadir, ${ }^{45}$ Shalini Sri Ranganathan, ${ }^{46}$ Saniya Sabzwari, ${ }^{26}$ Rohit Sarin, ${ }^{47}$ Manu Shankar-Hari, ${ }^{48}$ Michael Sharland, ${ }^{49}$ Yinzhong Shen, ${ }^{50}$ Joao Paulo Souza, ${ }^{51}$ Tshokey Tshokey, ${ }^{52}$ Sebastian Ugarte, ${ }^{53}$ Tim Uyeki, ${ }^{54}$ Sridhar Venkatapuram, ${ }^{48}$ Ablo Prudence Wachinou, ${ }^{55}$ Ananda Wijewickrama, ${ }^{56}$ Dubula Vuyiseka, ${ }^{57}$ Jacobus Preller, ${ }^{8,{ }^{*}}$ Romina Brignardello-Petersen, ${ }^{5,{ }^{*}}$ Elena Kum, ${ }^{5,}{ }^{*}$ Anila Qasim, ${ }^{5,}{ }^{*}$ Dena Zeraatkar, ${ }^{5,}{ }^{*}$

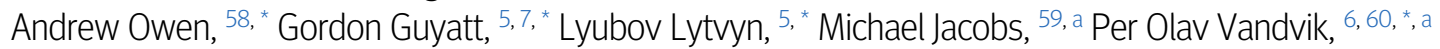
Janet Diaz ${ }^{8, *}$, a

\section{ABSTRACT}

\section{CLINICAL QUESTION}

What is the role of drugs in preventing covid-19? WHY DOES THIS MATTER?

There is widespread interest in whether drug interventions can be used for the prevention of covid-19, but there is uncertainty about which drugs, if any, are effective.

\section{RECOMMENDATIONS}

The second version of this living guideline reiterates the previous strong recommendation against the use of hydroxychloroquine and includes a new conditional recommendation against the use of tixagevimab-cilgavimab in individuals who do not have covid-19. HOW THIS GUIDELINE WAS CREATED

This living guideline is from the World Health Organization (WHO) and provides up to date covid-19 guidance to inform policy and practice worldwide. Magic Evidence Ecosystem Foundation (MAGIC) provides methodological support. A living systematic review with network analysis informs the recommendations. An international guideline development group (GDG) of content experts, clinicians, patients, an ethicist and methodologists produces recommendations following standards for trustworthy guideline development using the Grading of Recommendations Assessment, Development and Evaluation (GRADE) approach. UNDERSTANDING THE RECOMMENDATIONS

The living network meta-analysis informing this guideline included 12 trials (8379 participants) comparing hydroxychloroquine to standard care/placebo, and one trial (5197 participants) comparing tixagevimab-cilgavimab to standard care/placebo. When moving from evidence to the continued strong recommendation against the use of hydroxychloroquine, the GDG emphasised additional evidence suggesting no or little effect on mortality and hospital admission, and an increased risk of adverse effects. For the new conditional recommendation against the use of tixagevimab-cilgavimab, the GDG emphasised in vitro evidence reducing the applicability of available trial data. While trial results demonstrated modest reduction in the occurrence of laboratory confirmed symptomatic covid-19, lack of in vitro neutralisation of new SARS-CoV-2 sub-lineages was considered to have rendered these results obsolete. UPDATES

This is a living guideline. New recommendations will be published in this article and signposted by update notices to this guideline.

READERS NOTE

This is the second version of the living guideline for drugs to prevent covid-19. It complements the WHO living guideline on drugs to treat covid-19 and living guidance regarding covid-19 related clinical management. When citing this article, please consider adding the update number and date of access for clarity.

Drugs could be used as prophylaxis to prevent covid-19 developing in those who are free from disease. Such drugs complement vaccines that, through developing immune responses to SARS-Cov-2, reduce the risk of developing covid-19 and its consequences. Drugs to prevent covid-19 could target whole populations, those at higher risk of becoming infected with SARS-CoV-2 (due to their work, social circumstances, or a particular exposure), or those at higher risk of death and poor outcomes if infected. As of 6 February 2023, there are 14225 registered or ongoing trials investigating various drug interventions for covid-19 (see section on emerging evidence). ${ }^{1}$ This rapidly evolving evidence landscape requires trustworthy interpretation and expeditious clinical practice guidelines to inform clinicians, 
patients, governments, ministries, and health administrators.

This living guideline uses emerging evidence from randomised controlled trials (RCTs) on drugs to prevent covid-19 and complements the living WHO guideline on drugs to treat covid-19. ${ }^{2}$ The living network meta-analysis associated with this guideline incorporates new trial data and allows for analysis of comparative effectiveness. ${ }^{3}$ Details of the network meta-analysis and other related publications are listed in box 1 . We also use additional relevant evidence on long term safety, prognosis, and patient values and preferences related to covid-19 treatments to inform the living guidance.

\section{Box 1: Linked resources in this BMJ Rapid Recommendations cluster}

- Lamontagne F, Stegemann M, Agarwal A, et al. A living WHO guideline on drugs to prevent covid-19 [Version 2]. BMJ 2021;372:n526, doi:10.1136/bmj.n526

- World Health Organization. Drugs to prevent COVID-19: living guideline. 2023. https://apps.who.int/iris/bitstream/handle/10665/366530/WHO-2019-nCoV-prophylaxes-2023.1-eng.pdf

- MAGICapp (https://app.magicapp.org/\#/guideline/L6RxYL)

- Expanded version of the methods, processes, and results with multilayered recommendations, evidence summaries, and decision aids for use on all devices

- Bartoszko JJ, Siemieniuk R, Kum E, et al. Prophylaxis against covid-19: living systematic review and network meta-analysis [Version 2]. BM] 2021;373:n949, doi:10.1136/bmj.n949

- World Health Organization. Living guidance for clinical management of COVID-19. 2023. https://www.who.int/publications/i/item/WHO 2019-nCoV-clinical-2023.1

- Lamontagne F, Agarwal A, Rochwerg B, et al. A living WHO guideline on drugs for covid-19 [Version 13]. BMJ 2020;370:m3379, doi:10.1136/bmj.m3379

- World Health Organization. Therapeutics and COVID-19: Living guideline. 2023. https://www.who.int/publications/i/item/WHO2019-nCoV-therapeutics-2023.1

\section{What triggered this version of the guideline?}

The creation of a new recommendation on tixagevimab-cilgavimab prophylaxis followed the publication of one clinical trial suggesting potential benefits associated with the prophylactic use of these monoclonal antibodies and the subsequent emergence of in vitro evidence suggesting that these clinical trial data were potentially obsolete. ${ }^{4}$ For hydroxychloroquine, correspondence following the initial publication of the guideline justified re-examination of whether new evidence reinforced or contradicted the recommendation against hydroxychloroquine prophylaxis. ${ }^{5}$

\section{How to use this guideline}

This is a living guideline, so the recommendations included here will be updated, and new recommendations will be added for other prophylactic drugs for covid-19. The infographic provides a summary of the recommendations and includes links to MAGICapp for more details on the evidence and rationale for the recommendations. Box 2 outlines key methodological aspects of the guideline process.

\section{Box 2: How this living guideline was created (see MAGICapp for full} details https://app.magicapp.org/public/guideline/L6RxYL)

This guideline was developed by WHO and the MAGIC Evidence Ecosystem Foundation (MAGIC) with support from The BMJ. It was initiated due to an urgent need for trustworthy and living guidance to rapidly inform policy and practice worldwide during the covid-19 pandemic. WHO has partnered with MAGIC for their methodological support in the development and dissemination of living guidance for covid-19 drug treatments and prophylaxis, also published as BMJ Rapid Recommendations, to provide patients, clinicians, and policy makers with up to date, evidence based, and user-friendly guidelines.

Standards, methods, and processes for living and trustworthy guidance The guideline development group (GDG) produced the recommendations following standards for trustworthy guideline development using the GRADE (Grading of Recommendations Assessment, Development and Evaluation) approach, in compliance with the WHO Handbook for

Guideline Development 2nd Edition, ${ }^{6}$ the Institute of Medicine, and the Guideline International Network (G-I-N). ${ }^{7}$ Details are provided in the WHO guideline and MAGICapp (box 1).

\section{Selection and support of the GDG}

The methods chair (methodological expertise) and a clinical chair (content expertise) guided GDG discussions. Panel members were invited by WHO with the aim of achieving gender, geography, expertise, and patient representation balance in the GDG. No relevant conflict of interest was identified for any of the 34 GDG members.

The pre-selected clinical expert GDG, including patient partners, was convened most recently on 16 December 2022 to discuss methodological concepts relevant to guidelines for prophylactic interventions, review evidence summaries pertaining to hydroxychloroquine and tixagevimab-cilgavimab, and develop recommendations.

As recommended by the WHO handbook, the GDG aimed to create recommendations based on consensus but elected, at the beginning of the first GDG meeting, to call a vote if a consensus could not be reached. Before discussions started, the GDG determined that a simple majority would provide the direction of the recommendation and that $80 \%$ would be required to make a strong recommendation.

\section{Guideline perspective, outcomes, and values and preferences}

The target audience for this guidance consists primarily of clinicians and healthcare decision makers. The GDG considered an individual patient perspective but also took account of contextual factors (such as resources, feasibility, acceptability, equity) to accommodate a global context and the realities of different countries and healthcare systems. In the absence of empirical evidence on values and preferences guiding decisions for covid-19 prophylactic interventions, the GDG members relied on their own judgments of how well-informed individuals would value benefits, harms, and burdens of prophylactic interventions. The GDG concluded that:

- Mortality would be the outcome most important to individuals, followed by need for hospital admission, laboratory confirmed SARS-CoV-2 infection, and adverse effects leading to discontinuation.

- Most patients would be reluctant to use an intervention for which the evidence left high uncertainty regarding effects on outcomes they consider important, or when evidence suggested a low certainty of benefit. When beneficial effects, if present, would be very small, almost all patients would decline to use such an intervention.

- The GDG also considered optimal resource use at a public health level. The GDG placed a low value on allocating substantial resources for uncertain benefit and, conversely, a high value on preserving resources for interventions with a high certainty of benefit.

The GDG acknowledged, however, that values and preferences could vary. There may be individuals inclined to use a prophylactic intervention when an important benefit cannot be ruled out, particularly when the underlying condition is potentially fatal. On the other hand, other individuals will have a high threshold of likely benefit before opting to take medications if they are not yet ill. Although the GDG focused on an individual patient perspective, the members also considered a population perspective in which feasibility, acceptability, equity, and cost are important considerations, particularly when a very large number of otherwise healthy individuals might need to be treated before preventing one outcome.

\section{Sources of evidence}

To create recommendations, the GDG relied on evidence synthesised in a living network meta-analysis, as requested by the GDG. ${ }^{3}$ While the 
investigators responsible for the meta-analyses rated the certainty of the evidence, this was re-assessed independently by the GDG.

\section{Derivation of absolute effects for drug treatments}

The baseline risks were calculated from data from the control groups of trials included in the network meta-analysis, which also yielded the estimate of relative effects of prophylactic interventions. ${ }^{1}$ The evidence summaries that informed the guideline recommendation reported the anticipated absolute effects of hydroxychloroquine and

tixagevimab-cilgavimab compared with usual care across all patient-important outcomes, with explicit judgments of certainty in the evidence for each outcome. For mortality, the event rate among all participants randomised to standard care or placebo was used to calculate the baseline risk. For all other outcomes, the median event rate in the standard care or placebo arms was used, with each study weighted equally.

\section{Special considerations for recommendations on prophylaxis}

As detailed in the full WHO guideline (see box 1), the GDG considered the implications of very low risks of critical outcomes such as death in trials of prophylactic interventions. The GDG opted to consider the magnitude of effect (for example, trivial, small, moderate, or large effect) when rating certainty.

\section{Visual summary of recommendation}
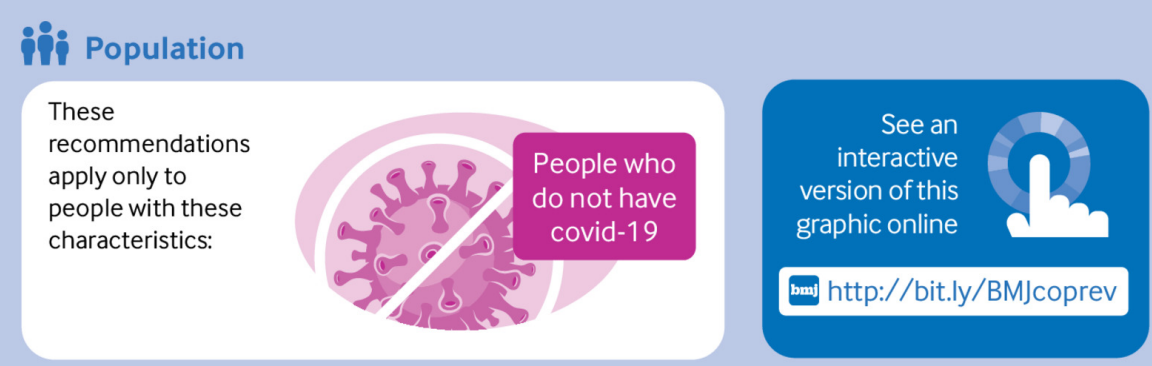

Recommendations

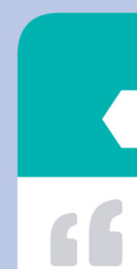

No intervention Strong Weak

We recommend not using hydroxychloroquine to prevent covid-19

Hydroxychloroquine

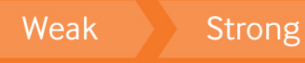

Evidence profile

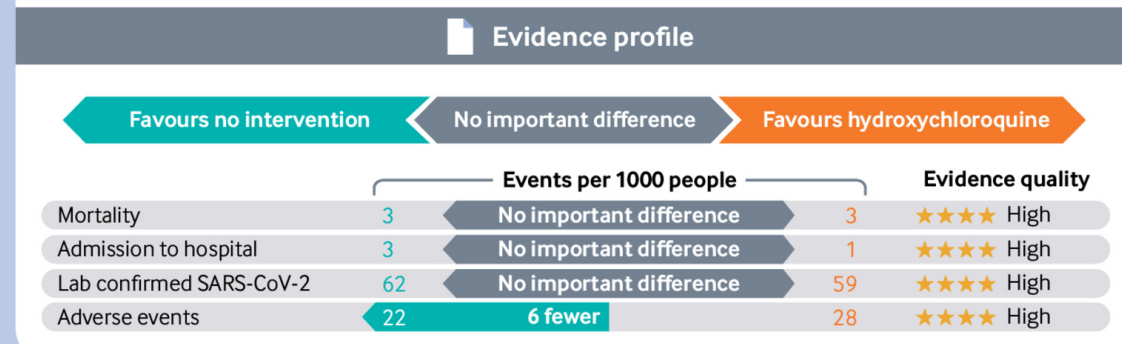

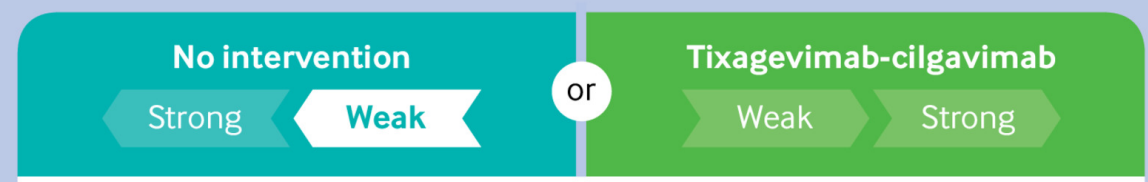

\section{We suggest not using tixagevimab-cilgavimab} to prevent covid-19

\begin{tabular}{|c|c|c|c|}
\hline \multicolumn{2}{|c|}{ Disclaimer } \\
\hline $\begin{array}{c}\text { Validation } \\
\text { This infographic is } \\
\text { not a validated } \\
\text { clinical decision aid }\end{array}$ & $\begin{array}{c}\text { This information is provided } \\
\text { without any representations, } \\
\text { conditions, or warranties that } \\
\text { it is accurate or up to date }\end{array}$ & $\begin{array}{c}\text { Responsibility } \\
\text { BMJ and its licensors assume no } \\
\text { responsibility for any aspect of } \\
\text { treatment administered with } \\
\text { the aid of this information }\end{array}$ & $\begin{array}{c}\text { Any reliance placed } \\
\text { on this information } \\
\text { is strictly at the } \\
\text { user's own risk }\end{array}$ \\
\hline
\end{tabular}

For the full disclaimer wording see BMJ's terms and conditions: http://www.bmj.com/company/legal-information/
Values and preferences

Hydroxychloroquine

The panel inferred that almost all well informed patients would not want to receive hydroxychloroquine given there are probably no positive effects and there was a risk of adverse events. The panel did not expect there would be much variation in values and preferences between patients for this intervention

Tixagevimab-cilgavimab

The panel inferred that, in the absence of compelling evidence of clinical

effectiveness for the currently circulating SARS-CoV-2 sublineages, the majority of informed individuals would not choose to receive tixagevimab-cilgavimab

\section{Justification}

Tixagevimab-cilgavimab

The panel reviewed in vitro evidence reducing the applicability of available trial data; while prophylactic use demonstrated modest reduction in laboratory-confirmed symptomatic covid-19, lack of in vitro neutralisation of new SARS-CoV-2 sub-lineages was considered to have rendered these results obsolete

\section{Conditions for use of treatment}

Tixagevimab-cilgavimab Any prophylactic use of this intervention should be restricted to extremely vulnerable individuals, and patients presenting a sublineage that is neutralised in-vitro.

Typical characteristics of extremely vulnerable individuals include:

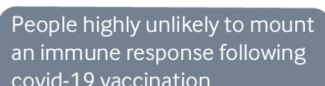
covid-19 vaccination

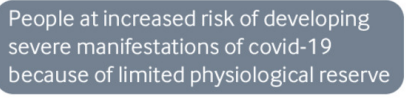




\section{Who do the recommendations apply to?}

This guideline applies to all individuals who do not have covid-19. For hydroxychloroquine, the GDG ultimately concluded that this recommendation was applicable irrespective of known exposure to individuals with SARS-CoV-2 infection or of different drug doses, based on pre-specified subgroup analyses as further outlined below.

In the case of tixagevimab-cilgavimab, the GDG concluded that prophylactic use of tixagevimab-cilgavimab should be strictly restricted to situations where there could be high certainty that the circulating sub-lineages exhibit in vitro neutralisation activity equivalent to those circulating in populations and regions when and where the supporting clinical trial was conducted. This decision was based on in vitro evidence suggesting lack of neutralisation efficacy for new circulating Omicron sub-lineages, and it applies to all individuals who do not have covid-19. The conditional recommendation acknowledges that a minority of high risk patients who have not been vaccinated or who are severely

immunocompromised may choose to receive

tixagevimab-cilgavimab. This exception would apply only where it could be established that such high risk patients were at risk of becoming infected with the SARS-CoV-2 sub-lineages that were prevalent during the trial.

\section{The guidance}

\section{Hydroxychloroquine}

Hydroxychloroquine is an immunomodulator that blocks toll-like receptors reducing dendritic cell activation. It is used to treat rheumatoid arthritis and systemic lupus erythematosus. It has an antiviral effect against many viruses in vitro, including SARS-CoV-2, but a clinically useful antiviral effect has not been shown for any viral infection.

The recommendation was informed by the linked systematic review and network meta-analysis that included 12 trials (8379 participants) randomising participants to hydroxychloroquine or to standard care/placebo. ${ }^{8-19}$ The trials included participants from North and South America and Europe who either had a known exposure to a person with SARS-CoV-2 infection or who were considered at risk given their professional occupations (such as healthcare workers). The most recent LNMA publication contains an additional trial comparing hydroxychloroquine to active interventions. ${ }^{320}$

Recommendation: We recommend against the use of hydroxychloroquine as prophylaxis in individuals who do not have covid-19 (strong recommendation).

\section{Understanding the recommendation}

Balance of benefits and harm-Used prophylactically, hydroxychloroquine has no or little effect on death and hospital admission (high certainty) and has no or little effect on laboratory confirmed SARS-CoV-2 infection (high certainty). It increases the risk of adverse effects leading to discontinuation of the drug (high certainty).

Subgroup effects-There were no subgroup effects according to known exposure to a person with SARS-CoV-2 infection or hydroxychloroquine dose regimen; extremely low event rates precluded investigation of subgroup effects for mortality. In the absence of subgroup effects, the GDG assumed similar relative effects across subgroups. As no subgroup effect modification was found, the strong recommendation is applicable across risk groups and dose regimens of hydroxychloroquine. Certain subgroups of vulnerable individuals, such as immunocompromised patients, were under-represented in previous hydroxychloroquine prophylaxis trials. However, the GDG maintained its view that, given the existing evidence, it would be extremely unlikely that large number of under-represented individuals would consent to participate in future hydroxychloroquine trials, and that these would find that hydroxychloroquine prophylaxis meaningfully reduces mortality reduction even in those subgroups.

Values and preferences-Applying the established values and preferences (box 2), the GDG inferred that almost all well-informed patients would decline hydroxychloroquine.

Applicability-Regarding special populations, none of the included RCTs enrolled children, and therefore the applicability of this recommendation to children is currently uncertain. However, as for other under-represented subgroups, the GDG had no reason to think that children would respond any differently to prophylactic hydroxychloroquine. There were similar considerations with regards to pregnant women, with no data directly examining this population, but no rationale to suggest they would respond differently to other adults. Hydroxychloroquine crosses the placental barrier, and there are concerns that it may lead to retinal damage in neonates.

Resource implications, feasibility, equity, and human rights-Hydroxychloroquine is relatively inexpensive and is widely available, including in low resource settings. Although the cost may be low per patient, the overall cost of delivering a prophylactic intervention on a large scale may be substantial. Moreover, the GDG raised concerns about diverting hydroxychloroquine stocks away from patients with other conditions for whom this medication is indicated. ${ }^{21}$

Uncertainties-The GDG acknowledged that a strong recommendation against hydroxychloroquine to prevent covid-19 indicates that this area is no longer a research priority and that resources devoted to clinical research should rather be oriented to evaluate more promising prophylactic interventions. The GDG felt that further research was unlikely to be acceptable to

under-represented subgroups or that it would uncover a subgroup of patients who would benefit from hydroxychloroquine prophylaxis on the most important outcomes (mortality, admission to hospital, and laboratory confirmed SARS-CoV-2 infection).

\section{Tixagevimab-cilgavimab}

Tixagevimab and cilgavimab are a combination (Evusheld, AZD7442) of human monoclonal antibodies that bind to non-overlapping regions for the receptor-binding domain of the SARS-CoV-2 Spike protein. Tixagevimab-cilgavimab is administered as a single intramuscular dose of $300 \mathrm{mg} .{ }^{22}$

The combination of tixagevimab and cilgavimab administered prophylactically as intravenous infusion prevented infection in animal models by ancestral SARS-CoV-2, leading to further interest in its use for this indication in clinical practice. However, animal data for currently circulating variants are unavailable, and evidence from in vitro neutralisation studies shows that for more recent variants (including BA.2.75.2, BQ.1, BQ.1.1, and XBB lineages) the in vitro neutralisation of both antibodies is compromised. The GDG concluded that this in vitro evidence rendered the clinical trial results obsolete.

Recommendation: We suggest not to use tixagevimab-cilgavimab in individuals who do not have covid-19 (conditional recommendation).

Understanding the recommendation-When moving from the evidence to the conditional recommendation against the use of 
tixagevimab-cilgavimab to prevent covid-19-related outcomes, the GDG emphasised in vitro evidence reducing the applicability of trial data. While clinical trial results suggested prophylactic use of tixagevimab-cilgavimab reduced the occurrence of laboratory confirmed covid-19, the GDG concluded that these modest benefits represented the best-case scenario obtained under lineages of SARS-CoV-2 that have since been replaced. Having concluded that recently emerged in vitro evidence rendered the clinical trial results obsolete, the GDG abstained from rating the certainty of the underlying evidence. A conditional recommendation was made recognising that, while it was extremely unlikely for previous SARS-CoV-2 sub-lineages to re-emerge, it nonetheless remained theoretically possible that former sub-lineages would continue to cause covid-19 in certain regions, and that, assuming that real-time monitoring of the prevalence of SARS-CoV-2 sub-lineages was available, a significant number of extremely vulnerable individuals may choose to receive prophylactic tixagevimab-cilgavimab. The GDG also considered that, although in vitro neutralisation is required for tixagevimab-cilgavimab to be considered for use as prophylaxis, it does not guarantee clinical effectiveness.

Applicability-While the recommendation applies to all individuals who do not have covid-19, a conditional recommendation implies that a minority may opt to receive this prophylactic intervention. Should this situation arise, all GDG members agreed that any prophylactic use of this intervention should be limited to a minority of patients who may be extremely vulnerable, in settings where prevailing SARS-CoV-2 sub-lineages were the same as the ones that caused covid-19 during the trial. Such patients include those who are highly unlikely to mount an immune response after covid-19 vaccination and who, due to a limited physiological reserve, are at high risk of developing severe manifestations of covid-19.

Balance of benefits and harms-See MAGICapp for a summary of findings from clinical trial data; this data ultimately did not directly bear on the recommendation because it was considered obsolete.

Values and preferences-The GDG inferred that, in the absence of compelling evidence of clinical effectiveness for the currently circulating SARS-CoV-2 sub-lineages, most informed individuals would not choose to receive tixagevimab-cilgavimab.

Resource implications, feasibility, equity, and human rights-The GDG placed a low value on potential benefits in regions where circulating SARS-CoV-2 sub-lineages demonstrate neutralisation activity, and considered that tixagevimab-cilgavimab is a costly intervention of limited availability and requiring parenteral administration. Conversely, the GDG placed a high value on preserving resources for interventions with a high certainty of benefit and noted the availability of effective therapeutic options for patients with covid-19.

Uncertainties-All members of the GDG acknowledged that the available in vitro data suggests that the results of the published clinical trials are now, at best, highly uncertain. However, the GDG was split regarding the strength of recommendation. This was driven by the certainty with which guideline users could rule out that the prevailing SARS-CoV-2 sub-lineages in their region would be resistant to tixagevimab-cilgavimab neutralisation. Those 14 panelists who voted for a strong recommendation pointed out that emerging sub-lineages tended to rapidly replace older sub-lineages all over the globe and that regional real-time monitoring of circulating SARS-CoV-2 sub-lineages was onerous and unrealistic in most geographical areas. Notwithstanding, those 17 panelists who voted for a conditional recommendation argued that, provided that these conditions could be satisfied, some highly vulnerable patients may choose to receive tixagevimab-cilgavimab.

The GDG inferred that restricting use of tixagevimab-cilgavimab to clinical trials would be futile considering the large number of participants required to demonstrate clinical effectiveness.

\section{Emerging evidence}

The unprecedented volume of planned and ongoing studies for covid-19 interventions implies that further evidence will emerge to inform policy and practice. ${ }^{1}$ An overview of registered and ongoing trials for covid-19 therapeutics and prophylaxis is available from the Infectious Diseases Data Observatory (through their living systematic review of covid-19 clinical trial registrations ${ }^{1}$ ), the WHO website, and other repositories such as the COVID-NMA initiative.

\section{How patients were involved in the creation of this article}

The guideline development GDG initially included four patient-partners who have had covid-19; with one taking active part in this update. Their perspectives were crucial in deciding on clinical questions and considering the values and preferences associated with drugs to prevent covid-19.

\section{AUTHOR AFFILIATIONS}

Université de Sherbrooke, Centre de recherche due CHU de Sherbrooke, Sherbrooke, Quebec, Canada

2

Charité - Universitätsmedizin Berlin, corporate member of Freie Universität Berlin and Humboldt-Universität zu Berlin, Department of Infectious Diseases, Respiratory Medicine and Critical Care, Berlin, Germany

Division of General Internal Medicine, McMaster University, Hamilton, Ontario, Canada

Division of General Internal Medicine \& Division of Clinical Epidemiology, University Hospitals of Geneva, Geneva, Switzerland

Department of Health Research Methods, Evidence and Impact, McMaster University, Hamilton, Ontario, Canada

6

MAGIC Evidence Ecosystem Foundation, Oslo, Norway

Department of Medicine, McMaster University, Hamilton, Ontario, Canada

8 World Health Organization, Geneva, Switzerland London, UK

10 Communicable Disease Center, Qatar

11 Ministry of Health and Population, Cairo, Egypt

12 Departamento de Materno Infantil, Universidade Federal Fluminense, Brazil Antioquia University Medellín, Colombia

14 Geneva University Hospital, Switzerland

15 Infection Division, Department of Pulmonology and Respiratory Medicine, Faculty of Medicine Universitas Indonesia

Department of Anesthesia and Intensive Care Medicine, Humanitas Clinical and Research Center-IRCCS, Rozzano, Italy

17 Division of Critical Care Medicine at Christian Medical College, Vellore, India 18 Adult Infectious Disease Centre, University Teaching Hospital, Lusaka, Zambia 19 Department of Infectious Diseases, Hanoi Medical University, Hanoi, Vietnam 
20

Peking Union Medical College Hospital, Beijing, China

21

Division of Emergency Medicine, Faculty of Medicine and Health Sciences, Stellenbosch University, Cape Town, South Africa

22 USA

23

Ministry of Health, Afghanistan

24

Royal Melbourne Hospital, Melbourne, Australia

25

Ziauddin University, Karachi, Pakistan

26

Aga Khan University, Karachi, Pakistan

27

Stanley Ho Centre for Emerging Infectious Diseases, Chinese University of Hong Kong, Hong Kong SAR, China

28

Guy's \& St Thomas' NHS Foundation Trust, London, UK

29

Indira Gandhi Memorial Hospital, Maldives

30

All India Institute of Medical Sciences, New Delhi, India

31

McMaster University (alumnus)

32

Pulmonary Division, Heart Institute (InCor)- HCFMUSP, Medical School, University of Sao Paulo, São Paulo, Brazil and Research Institute, Hospital do Coração (HCor), São Paulo, Brazil

33 Sungkyunkwan University School of Medicine, Samsung Medical Center, Seoul, Republic of Korea

34

Department of Paediatrics and Emergency Medicine, University of British Columbia, Vancouver, Canada

35

St George's University of London, UK

36 Department of Anaesthesia and Critical Care, College of Health Sciences, Makerere University, Kampala, Uganda

37

Coraçao Hospital, São Paulo, Brazil

38

National Center for Infectious Diseases, Singapore

39

Pangaea Harare, Zimbabwe

40 Emergency Medical Services, Faculty of Medicine, Tunis, Tunisia

41

Pulmonology, Istituti Clinici Scientifii Maygeri, Italy

42

Alcivar Hospital, Guayaquil, Ecuador

43

Sheikh Shakhbout Medical City, Abu Dhabi, UAE

44

Central Research Institute of Epidemiology of Rospotrebnadzor, Moscow, Russia

45

Division of Pulmonary and Critical Care Medicine, David Geffen School of Medicine,

University of California Los Angeles, Los Angeles, California, USA

46

University of Colombo, Colombo, Sri Lanka

47

National Institute of Tuberculosis and Respiratory Diseases, New Delhi, India

King's College, London, UK

49

St. George’s University Hospital, London, UK

50

Shanghai Public Health Clinical Center, Fudan University, Shanghai, China

University of Sao Paulo, Sao Paulo, Brazil
52

JDW National Referral Hospital, Bhutan

53

Faculty of Medicine Andres Bello University, Indisa Clinic, Santiago, Chile

54

Influenza Division, Centers for Disease Control and Prevention, USA

55

National Hospital for Tuberculosis and Pulmonary Diseases, Benin

56

Ministry of Health, Sri Lanka

57

University of Stellenbosch, South Africa

58

University of Liverpool, Liverpool, UK

Royal Free London NHS Foundation Trust, London, UK

60

Department of Health Economics and Health Management, Institute for Health and Society, University of Oslo, Oslo, Norway

Not guideline development group member; resource for methodology, systematic review, and content support

a

co-senior author

Funding: Bill \& Melinda Gates Foundation, UNITAID, Norwegian Directorate of Public Health and Germany provided funding for this guideline, with MAGIC providing pro-bono contributions and support to WHO in the context of the covid-19 pandemic.

Competing interests: All guideline GDG members have completed the WHO interest disclosure form. All authors have completed the BMJ Rapid Recommendations interest of disclosure form. The WHO, MAGIC, and The BMJ judged that no GDG member had any financial conflict of interest. Professional and academic interests are minimised as much as possible, while maintaining necessary expertise on the GDG to make fully informed decisions. MAGIC and TheBM/assessed declared interests from other co-authors of this publication and found no conflicts of interests

Provenance and peer review: This publication was commissioned by The BM/in partnership with WHO and the MAGIC Evidence Ecosystem Foundation, in the context of the BMJ Rapid Recommendations. Pre-publication internal and external peer-review was managed by WHO, and internal review at The $B M J$. Post-publication review through rapid responses on bmj.com and through MAGICapp.

We thank all the following collaborators who contributed to this endeavour, as detailed in the WHO guidance (see link in box 1)

- World Health Organisation (WHO) Secretariat for Therapeutics and COVID-19

- External reviewers for WHO

We also thank

- The living systematic review and network meta-analysis team, led by investigators Reed Siemienuk and Romina Brignardello-Petersen at McMaster University, Canada

- BMJ editorial team with Tom Nolan (head of education), Will Stahl-Timmins (data graphics designer) and Greg Cotton (technical editor) of this living guideline publication in The BMJ

- Alistair MacLean, Brittany Maguire, Philippe Guerin, and Sumayyah Rashan for providing an overview of registered and ongoing trials, from the Infectious Diseases Data Observatory (IDDO) living systematic review for covid-19 clinical trial registration (https://www.iddo.org/research-themes/covid-19/ive-systematic-clinical-trial-review).

- Andrew Owen (University of Liverpool, UK) for contributions on pharmacological considerations and analyses

1 Maguire BJ, Guérin PJ. A living systematic review protocol for COVID-19 clinical trial registrations. Wellcome Open Res 2020;5:. doi: 10.12688/wellcomeopenres.15821.1 pmid: 32292826

2 Siemieniuk R, Rochwerg B, Agoritsas T, etal. A living WHO guideline on drugs for covid-19. BM 2020;370:. pmid: 32887691

3 Bartoszko Jl, Siemieniuk RAC, Kum E, etal. Prophylaxis against covid-19: living systematic review and network meta-analysis. BM/2021;373:. doi: 10.1136/bmj.n949 pmid: 33903131

4 Imai M, Ito M, Kiso M, etal. Efficacy of antiviral agents against omicron subvariants BQ.1.1 and XBB. N Engl / Med 2023;388:-91. doi: 10.1056/NEJMc2214302 pmid: 36476720

5 Schilling WH, Callery JJ, Chandna A, Hamers RL, Watson JA, White NJ. The WHO guideline on drugs to prevent COVID-19: small numbers- big conclusions. Wellcome Open Res 2021;6: doi: 10.12688/wellcomeopenres.16741.1 pmid: 34395925 
6 World Health Organization. Handbook for guideline development. 2014. https://apps,who.int/iris/handle/10665/145714.

7 Qaseem A, Forland F, Macbeth F, Ollenschläger G, Phillips S, van der Wees PBoard of Trustees of the Guidelines International Network. Guidelines International Network: toward international standards for clinical practice guidelines. Ann Intern Med 2012;156:-31. doi: 10.7326/0003-4819-156-7-201204030-00009 pmid: 22473437

8 Abella BS, Jolkovsky EL, Biney BT, etalPrevention and Treatment of COVID-19 With Hydroxychloroquine (PATCH) Investigators. Efficacy and safety of hydroxychloroquine vs placebo for pre-exposure SARS-CoV-2 prophylaxis among health care workers: a randomized clinical trial. JAMA Intern Med 2021;181:-202. doi: 10.1001/jamainternmed.2020.6319 pmid: 33001138

9 Barnabas RV, Brown ER, Bershteyn A, etal. Hydroxychloroquine as postexposure prophylaxis to prevent severe acute respiratory syndrome coronavirus 2 infection: a randomized trial. Ann Intern Med 2020; pmid: 33284679

10 Boulware DR, Pullen MF, Bangdiwala AS, etal. A randomized trial of hydroxychloroquine as postexposure prophylaxis for Covid-19. N Engl J Med 2020;383:-25. doi: 10.1056/NE/Moa2016638 pmid: 32492293

11 Grau-Pujol B, Camprubi-Ferrer D, Marti-Soler H, etal. Pre-exposure prophylaxis with hydroxychloroquine for COVID-19: a double-blind, placebo-controlled randomized clinical trial. Trials 2021;22:. doi: 10.1186/s13063-021-05758-9 pmid: 34781981

12 McKinnon JE, Wang DD, Zervos M, etal. Safety and tolerability of hydroxychloroquine in health care workers and first responders for the prevention of COVID-19: WHIP COVID-19 Study. Int I Infect Dis 2022;116:-73. doi: 10.1016/i.i.jid.2021.12.343 pmid: 34954095

13 Mitjà O, Corbacho-Monné M, Ubals M, etalBCN-PEP-CoV2 Research Group. A cluster-randomized trial of hydroxychloroquine for prevention of Covid-19. N Engl/ Med 2021;384:-27. doi: 10.1056/NEJMoa2021801 pmid: 33289973

14 Naggie S, Milstone A, Castro M, etalHERO Research Program. Hydroxychloroquine for pre-exposure prophylaxis of COVID-19 in health care workers: a randomized, multicenter, placebo-controlled trial Healthcare Worker Exposure Response and Outcomes of Hydroxychloroquine (HERO-HCQ). Int I Infect Dis 2023:129:-8. doi: 10.1016/j.i.jid.2023.01.019 pmid: 36682681

15 Polo R, García-Albéniz X, Terán C, etalEPICOS. Daily tenofovir disoproxil fumarate/emtricitabine and hydroxychloroquine for pre-exposure prophylaxis of COVID-19: a double-blind placebo-controlled randomized trial in healthcare workers. Clin Microbiol Infect 2023;29:-93. doi: 10.1016/j.cmi.2022.07.006 pmid: 35940567

16 Rajasingham R, Bangdiwala AS, Nicol MR, etal. Hydroxychloroquine as pre-exposure prophylaxis for COVID-19 in healthcare workers: a randomized trial. Clin Infect Dis 2020;ciaa1571. .pmid: 33068425

17 Rojas-Serrano J, Portillo-Vásquez AM, Thirion-Romero I, etal. Hydroxychloroquine for prophylaxis of COVID-19 in health workers: A randomized clinical trial. PLoS One 2022;17:e0261980. doi: 10.1371/journal.pone.0261980 pmid: 35139097

18 Syed F, Hassan M, Arif MA, etal. Pre-exposure prophylaxis with various doses of hydroxychloroquine among healthcare personnel with high-risk exposure to COVID-19: a randomized controlled trial. Cureus 2021;13:e20572. doi: 10.7759/cureus.20572 pmid: 35103151

19 Tirupakuzhi Vijayaraghavan BK, Jha V, Rajbhandari D, etalHOPE Investigators. Hydroxychloroquine plus personal protective equipment versus personal protective equipment alone for the prevention of laboratory-confirmed COVID-19 infections among healthcare workers: a multicentre, parallel-group randomised controlled trial from India. BMJ Open 2022;12:e059540. doi: 10.1136/bmjopen-2021-059540 pmid: 35649613

20 Seet RCS, Quek AML, Ooi DSQ, etal. Positive impact of oral hydroxychloroquine and povidone-iodine throat spray for COVID-19 prophylaxis: An open-label randomized trial. Int J Infect Dis 2021;106:-22. doi: 10.1016/i.jij.2021.04.035 pmid: 33864917

21 Schreiber K, Sciascia S, Bruce IN, etal. Hydroxychloroquine in patients with rheumatic diseases during the COVID-19 pandemic: a letter to clinicians. Lancet Rheumatol 2020;2:-6. doi: 10.1016/S2665-9913(20)30382-9 pmid: 33163976

22 Levin MJ. Ustianowski A, De Wit S, etalPROVENT Study Group. Intramuscular AZD7442 (Tixagevimab-cilgavimab) for prevention of covid-19. N Engl/ Med 2022;386:-200. doi: 10.1056/NEJMoa2116620 pmid: 35443106

\section{Main infographic: Summary of recommendations and evidence}

This article is made freely available for personal use in accordance with BMJ's website terms and conditions for the duration of the covid-19 pandemic or until otherwise determined by BMJ. You may download and print the article for any lawful, non-commercial purpose (including text and data mining) provided that all copyright notices and trade marks are retained. 